\title{
Contemporary radiation therapy techniques in malignant pleural mesothelioma: A case report
}

Joshua Noah Halpern, Shauna Marie McVorran, Mordecha Loksen

The Brooklyn Hospital Center, United States

Received: September 30, 2015

DOI: $10.5430 /$ jst.v6n1p54
Accepted: November 27, 2015 Online Published: December 23, 2015

URL: http://dx.doi.org/10.5430/jst.v6n1p54

\begin{abstract}
Malignant pleural mesothelioma (MPM) is a rare but very aggressive neoplasm. Prognosis is poor and the disease is fatal within 4-8 months without treatment. Even with a trimodality treatment approach, which has been shown to produce significant morbidity, median survival does not exceed 11 months. There is a need therefore for exploring new treatment options that can improve both qualitative and quantitative outcomes.

This case report describes locally advanced malignant pleural mesothelioma treated with a unique, customized irradiation technique involving a combination of 3D conformal therapy and intensity modulated radiotherapy (IMRT). This allowed for treatment of the pleura to a high dose, while sparing the lung parenchyma. In this patient, our approach resulted in a prolonged remission. It is our hope that this stimulates new interest in the use of modern day radiation techniques in the treatment of MPM.
\end{abstract}

Key Words: Malignant pleural mesothelioma, Treatment, 3D conformal therapy, Intensity modulated radiotherapy

\section{INTRODUCTION}

Mesothelioma is a neoplasm that arises from the mesenchymal cells that form the lining of most body cavities. It typically involves the pleura (87\%) but may also affect the peritoneum $(5.1 \%)$, pericardium $(0.4 \%)$ and, more rarely, the testes. Approximately 2,500 cases are diagnosed each year in the United States. Malignant pleural mesothelioma (MPM), the most common clinical subtype, is strongly linked to asbestos exposure and may occur many decades after the initial exposure. ${ }^{[1]}$

Without treatment, MPM is fatal within 4-8 months, with death usually occurring as a result of infection or respiratory failure following progression of the disease. Current guidelines suggest surgery and chemotherapy to be the standard of care in the treatment of this neoplasm. ${ }^{[2]}$ The role of radiation in the management of malignant pleural mesothelioma has not been well defined, but it is generally recommended as adjuvant or palliative therapy. ${ }^{[3,4]}$ With the recent introduction of trimodality treatment, involving induction chemotherapy, definitive surgery and adjuvant radiation, patients have survived as long as 5 years, but median survival does not exceed 11 months. ${ }^{[5,6]}$

The use of contemporary radiation techniques as sole treatment for MPM is poorly documented. We therefore present here a case of locally advanced malignant mesothelioma, treated with a unique, customized irradiation technique. This involved both 3D conformal therapy and intensity modulated radiotherapy (IMRT) and resulted in a prolonged remission.

\section{Case presentation}

The patient was an 89-year-old male with history of hypertension, initially evaluated for progressive shortness of breath

*Correspondence: Joshua Noah Halpern; Email: jhalpern01@ yahoo.com; Address: The Brooklyn Hospital Center, United States. 
and $20 \mathrm{lb}$ weight loss. He was a long term smoker and had previously been employed as a sanitation worker and in a dry cleaning store. He denied any obvious exposure to asbestos. A chest X-ray showed a right-sided pleural effusion and a subsequent CT scan confirmed a loculated pleural effusion with a small focus of pleural thickening on the right side (see Figure 1).

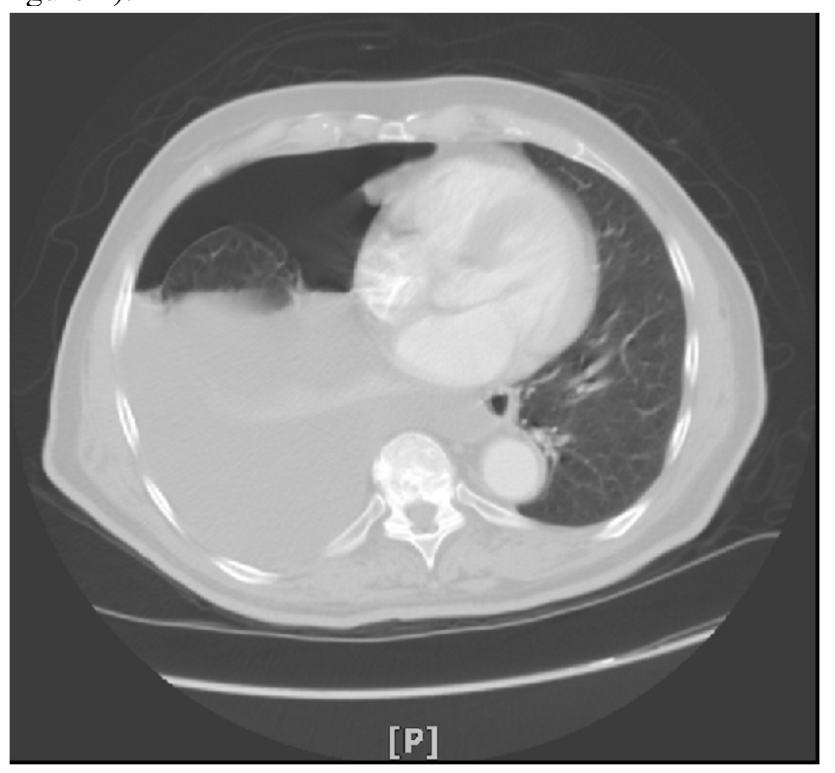

Figure 1. Computerized tomography scan of the chest showing a large right-sided pleural effusion associated with minimal pleural enhancement.There is some mass effect and shift of midline to the left. An underlying mass or infiltrate could not be excluded. There are also soft tissue densities in the pre-carinal space measuring $1.7 \mathrm{~cm} \times 1.3 \mathrm{~cm}$ and in the sub-carinal space measuring $2.3 \mathrm{~cm} \times 1.3 \mathrm{~cm}$, consistent with lymph nodes.

The patient underwent thoracocentesis and the resulting cytology was positive for malignant cells. He then had a thoracoscopy with biopsy of pleural and parenchymal nodules which revealed malignant mesothelioma. 5/5 lymph nodes were negative for malignant findings. Staging PET/CT showed a large hypermetabolic focus along the lateral and inferior pleural surface of the right thorax only, with no distant metastasis. As a result of this, the patient was diagnosed with Stage II (T2 N0 M0) malignant pleural mesothelioma.

The tumor was deemed unresectable. The patient received 4 doses of pemetrexed $\left(500 \mathrm{mg} / \mathrm{m}^{2}\right)$ without a significant response. He was considered for chemotherapy with cisplatin but was not a candidate due to poor renal function. He was then referred for palliative radiation therapy

\subsection{Radiation therapy}

A complex 8 field arrangement was utilized (see Figures 2 and 3). The mediastinum and the right pleural field were treated with penetrating photon beams (10 MV) via IMRT techniques, while the central fields, covering the lung parenchyma, were treated with superficially distributed electron beams ( 9 and $12 \mathrm{MeV}$ ) according to the thickness of the tissue. As shown in Figure 2, the electron/photon junction was feathered by shifting $1 \mathrm{~cm}$ medially and laterally every $18 \mathrm{~Gy}$. He received a total of $66.6 \mathrm{~Gy}$ in 37 fractions.

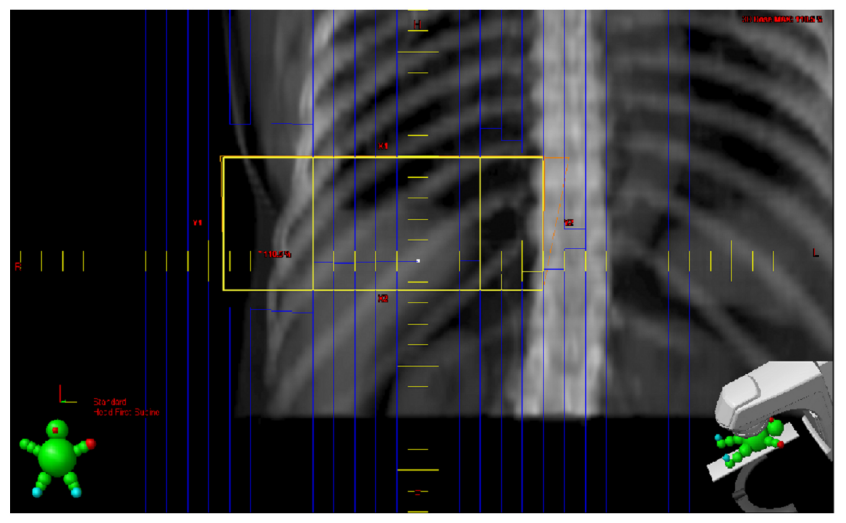

Figure 2. Field arrangement. Lateral and medial portions of the fields were treated anteriorly and posteriorly with a 10 MV photon beam energy, while the central portion was treated with superficially penetrating electron beams ( 9 and $12 \mathrm{MeV}$ ) in order to spare the lung and liver parenchyma. The electron/photon junction was shifted $1 \mathrm{~cm}$ every $18 \mathrm{~Gy}$.

The Dose Volume Histogram (DVH) of the IMRT treatment plan can be seen in Figure 4. It indicates an under dose of the GTV which was compensated for with electron treatments as per the tabulated electron dose distribution. The total projected GTV coverage, including electrons, was $90 \%$ of the prescribed dose.

\subsection{Outcomes}

The patient tolerated radiation therapy well with no adverse events. He was seen 1 month post treatment and thereafter at 3-month intervals. Clinically, the patient reported significant improvement in his shortness of breath and other symptoms. A chest CT obtained 2 months post treatment revealed a significant improvement in the tumor bulk in the upper portion of the pleural space but worsening interstitial opacification of the right lower lobe consistent with radiation changes. Another scan, obtained 11 months post treatment (see Figure $5)$, showed no interval change in the right pleural thickening, right lower lobe consolidation or associated adenopathy. The pleural effusion did not recur. Unfortunately the patient passed away soon thereafter, 21 months after diagnosis was confirmed and 15 months after completion of palliative radiation therapy. Cause of death was unrelated to his malignancy. 


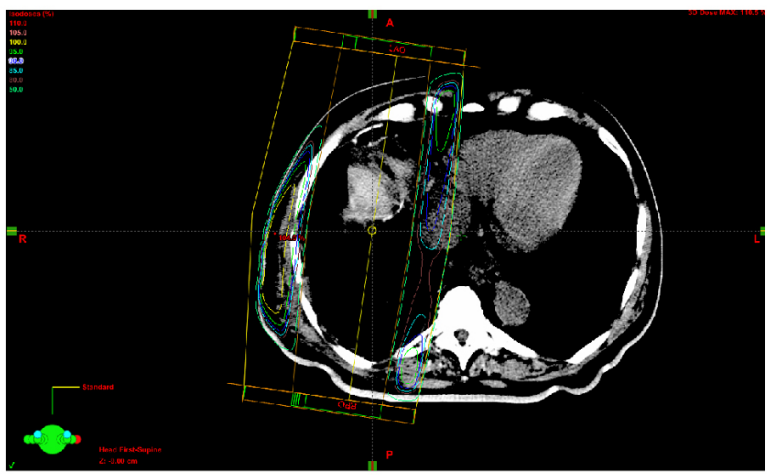

a

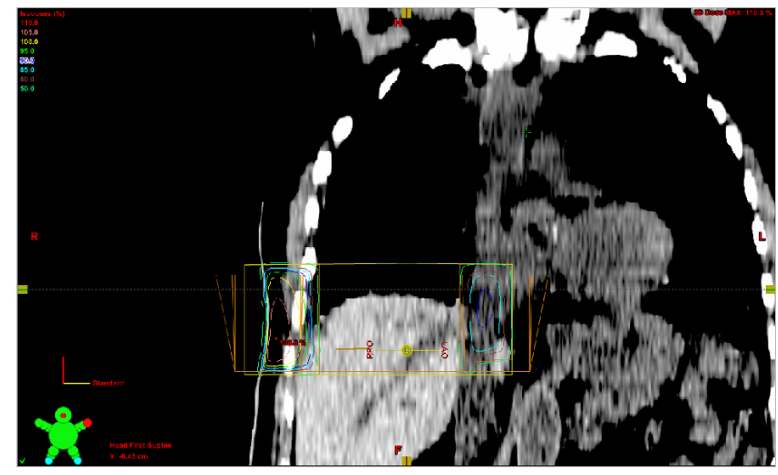

b

Figure 3. Treatment setup for the oblique AP/PA beams in a) transverse view and b) coronal section. Isodose distributions are displayed. The middle of the field is treated with electrons in order to spare the deeper structures, including lung and liver parenchyma.

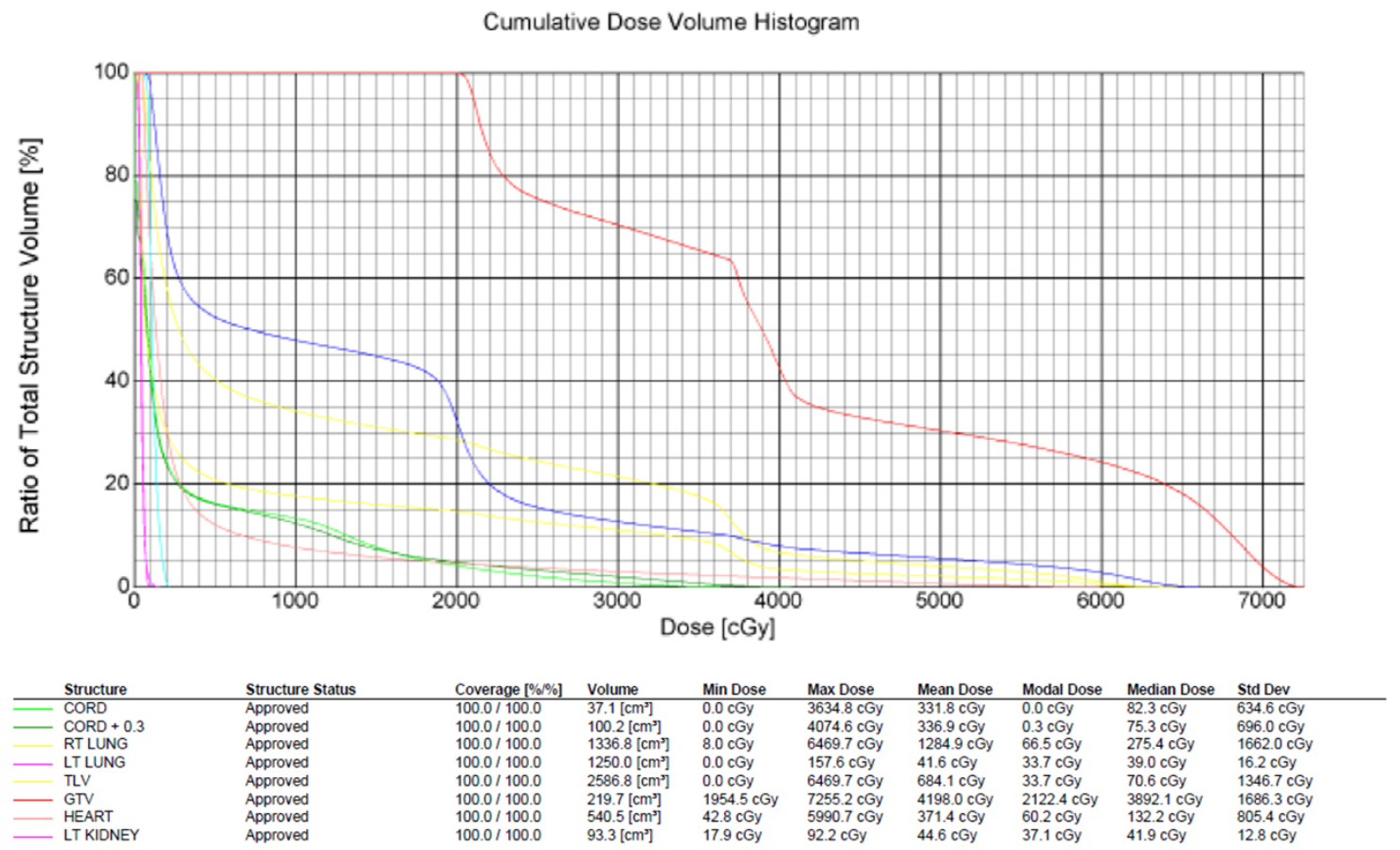

Figure 4. Dose volume histogram (DVH) of the GTV and of vital structure including liver, lung, cord and heart

\section{DiscuSSION}

MPM is a rare but very aggressive neoplasm. There are three histological subtypes: epithelioid, sarcomatoid and biphasic (mixed). Tumor histology, along with age at diagnosis, performance status and the presence of weight loss, is strongly correlated with prognosis: epithelioid mesothelioma carries a better prognosis than the sarcomatoid or mixed histology. ${ }^{[7]}$ Regardless, prognosis of MPM is poor and the disease is fatal within 4-8 months without treatment.

Current guidelines suggest treatment of MPM to be palliative or potentially curative depending on the cancer stage. ${ }^{[2]} \mathrm{Pa}-$ tients diagnosed at a stage at which potentially curative treatments are not effective may instead receive palliative treatments to relieve pain and improve quality of life. Chemotherapy may be given to medically operable patients with stage I-III MPM, either before or after surgery. Chemotherapy alone is recommended for medically inoperable patients with stages I-IV MPM and in those with sarcomatoid histology. ${ }^{[8,9]}$

Surgery and radiotherapy have a very limited role in the treatment of patients with advanced MPM and chemotherapy is the only potential treatment option for the majority of people 
diagnosed with mesothelioma. ${ }^{[10]}$ A trimodality therapeutic approach, utilizing chemotherapy, surgery and hemithoracic radiotherapy, has recently been proposed for the treatment of MPM, with good preliminary results. ${ }^{[11-13]}$ The median survival was reported as 11 months. The survival rate however, is influenced by nodal status and response to chemotherapy.

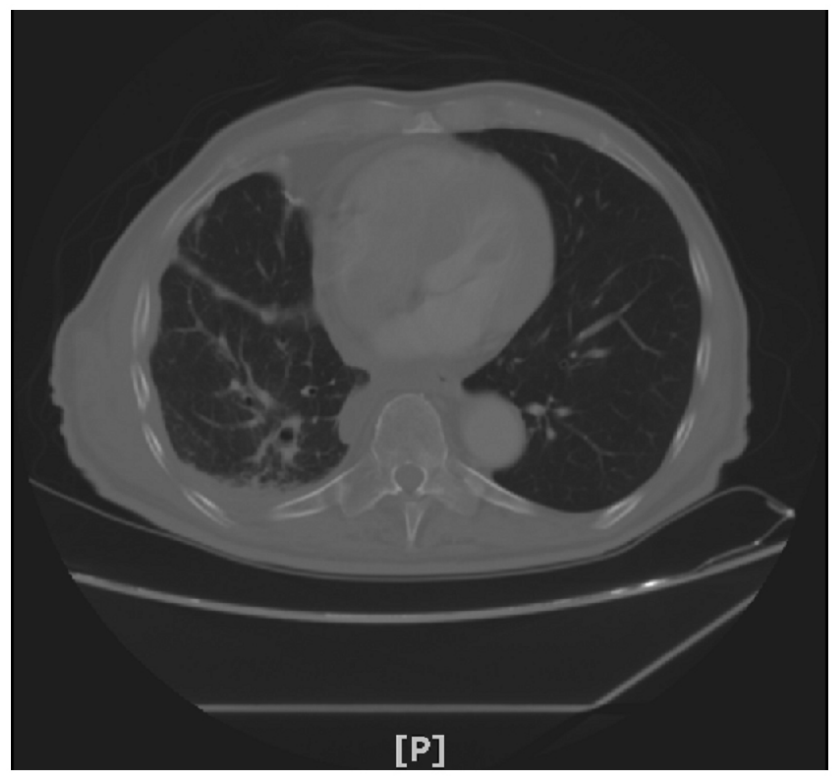

Figure 5. Computerized tomography scan of the chest 11 months post radiation treatment. Remarkable for residual findings around the right pleura which may represent persistent disease or post radiation fibrotic changes. No chest wall invasion is identified.

The response of MPM to radiotherapy has been very modest as the high doses required would be poorly tolerated by the adjacent vital tissues, particularly the lung parenchyma. However, postsurgical radiotherapy has been proven effective. The most recent and effective radiotherapy technique used is intensity modulated radiotherapy (IMRT). IMRT has the potential to cover the dose related concerns and thus has been applied with no toxicity in patients following extra pleural pneumonectomy. The combined use of radiotherapy and extra pleural pneumonectomy has proven successful in managing local tumor recurrence; however patients tend to die of metastatic disease. ${ }^{[4]}$

There is a lack of published evidence on the use of contemporary radiation techniques in unresected, locally advanced mesothelioma. One of the challenges for individualized radiation in this kind of tumor is that the target field is large and surrounds almost the entire lung. Ung et al. (2006), ${ }^{[10]}$ in their systematic review of the literature on this topic, concluded that hemithoracic radiation, without definitive surgery, results in significant toxicity including radiation pneumonitis, lung fibrosis and bronchopleural fistula, without any survival benefit. In their series however, treatment did not spare the lung parenchyma. They concluded that based on the lack of evidence for the use of radical radiation therapy alone in the management of patients with MPM, radical radiation should not be offered as a curative treatment option to patients with MPM. ${ }^{[10]}$

In the patient described in this case study, a relatively higher dose of radiation (66.6 Gy vs. $50 \mathrm{~Gy}$ ) was used, with a unique method of sparing intrathoracic organs, in our case both lung and liver. This technique allowed us to achieve a significant clinical response. Also, with this approach, radiation doses to other vital structures, such as liver and lung were kept to a minimum, thus reducing toxicity significantly. Close follow up to 11 months after completion of radiation therapy did not show any recurrence. Our case, and a few recent published reports, ${ }^{[14]}$ indicates promising imaging and clinical response with modern day radiation in locally advanced pleural mesothelioma. Attention should be paid however, to the radiation dose to the lung parenchyma and other vital structures. More studies will be needed for further documentation of the benefit of radiation in this condition. We hope, however, that this report will stimulate more use of radiation therapy in the treatment of MPM.

\section{CONFlicts of InTEREST Disclosure}

The author declares that there is no conflict of interest statement.

\section{REFERENCES}

[1] Hodgson JT, Darnton A. The quantitative risks of mesothelioma and lung cancer in relation to asbestos exposure. Ann Occup Hyg. 2000 Dec; 44(8): 565-601. PMid:11108782. http://dx.doi.org/10. 1093/annhyg/44.8.565

[2] National Comprehensive Cancer Network (NCCN). NCCN Clinical Practice Guidelines in Oncology. Malignant Pleural Mesothelioma Version 1.2015. National Comprehensive Cancer Network. Available from: http://www.nccn.org/professionals/physi cian_gls/f_guidelines.asp\#site

[3] Rusch VW, Rosenzweig K, Venkatraman E, et al. A phase II trial of surgical resection and adjuvant high-dose hemithoracic radiation for malignant pleural mesothelioma. J Thorac Cardiovasc Surg. 2001; 122: 788-95. PMid:11581615. http://dx.doi.org/10.1067/m tc. 2001.116560

[4] Yajnik S, Rosenzweig KE, Mychalczak B, et al. Hemithoracic radiation after extrapleural pneumonectomy for malignant pleural mesothelioma. J Radiat Oncol Biol Phys. 2003; 56: 1319-26. 
http://dx.doi .org/10.1016/S0360-3016(03)00287-6

[5] Sugarbaker DJ, Garcia JP, Richards WG, et al. Extrapleural pneumonectomy in the multimodality therapy of malignant pleural mesothelioma. Results in 120 consecutive patients. Ann Surg. 1996 Sep; 224(3): 288-94. PMid:8813257. http://dx.doi.org/10.10 97/00000658-199609000-00005

[6] Huncharek M, Kelsey K, Mark EJ, et al. Treatment and survival in diffuse malignant pleural mesothelioma: a study of 83 cases from the Massachusetts General Hospital. Anticancer Res. 1996 May-Jun; 16(3A): 1265-8. PMid:8702248.

[7] Travis WD, Brambilla E, Muller-Hermelink HK, et al. Pathology and Genetics of Tumours of the Lung, Pleura, Thymus and Heart. Lyon: IARC Press. Chapter 2: Tumors of the pleura. 2004: 25-144.

[8] Kelly RJ, Sharon E, Hassan R. Chemotherapy and targeted therapies for unresectable malignant mesothelioma. Lung Cancer. 2011; 73: 256-63. PMid:21620512. http://dx.doi.org/10.1016/j.lun gcan.2011.04.014

[9] Ellis P, Davies AM, Evans WK, et al. The use of chemotherapy in patients with advanced malignant pleural mesothelioma: a systematic review and practice guideline. J Thorac Oncol. 2006; 1: 591-601. http://dx.doi .org/10.1016/S1556-0864(15)30366-X

[10] Ung YC, Yu E, Falkson C, et al. The Role of Radiation Therapy in Malignant Pleural Mesothelioma: A Systematic Review. The Lung
Cancer Disease Site Group. Report Date: February 6, 2006. Available from: https://www. cancercare.on.ca/cms/one.aspx?obje ct Id $=10286 \&$ context $I d=1377$

[11] Krug LM, Pass HI, Rusch VW, et al. Multicenter phase II trial of neoadjuvant pemetrexed plus cisplatin followed by extrapleural pneumonectomy and radiation for malignant pleural mesothelioma. J Clin Oncol. 2009; 27: 3007-13. PMid:19364962. http: //dx.doi.org/10.1200/JC0.2008.20.3943

[12] DePerrot M, Feld R, Cho BC, et al. Trimodality therapy with induction chemotherapy followed by extrapleural pneumonectomy and adjuvant high-dose hemithoracic radiation for malignant pleural mesothelioma. J Clin Oncol. 2009; 27: 1413-8. PMid:19224855. http://dx.doi.org/10.1200/JC0.2008.17.5604

[13] Bolukbas S, Manegold C, Eberlein M, et al. Survival after trimodality therapy for malignant pleural mesothelioma: radical pleurectomy, chemotherapy with cisplatin/permetrexed and radiotherapy. Lung Cancer. 2011; 71: 75-81. PMid:19765853. http://dx.doi.org/1 $0.1016 / \mathrm{j}$. lungcan.2009.08.019

[14] Chan MF, Chui CS, Song Y, et al. A novel radiation therapy technique for malignant pleural mesothelioma combining electrons with intensity-modulated photons. Radiother Oncol. 2006; 79: 21823. PMid:16698098. http://dx.doi.org/10.1016/j.radonc. 2006.04 .007 